\section{MS9-P13 Structural studies of new $\beta$-lactoglobulin variants possessing modifications introduced to the binding site}

\author{
Joanna I. Loch ${ }^{1}$, Magdalena Tworzydło ${ }^{2}$, Piotr Bonarek ${ }^{2}$, \\ Agnieszka Polit ${ }^{2}$, Kinga Kaczor ${ }^{1}$, Monika Siuda ${ }^{1}$, Mateusz \\ Gotkowski ${ }^{1}$, Krzysztof Lewinski
}

1. Faculty of Chemistry, Jagiellonian University in Kraków, Poland 2. Faculty of Biochemistry, Biophysics and Biotechnology, Jagiellonian University in Kraków, Poland

email: loch@chemia.uj.edu.pl

Bovine $\beta$-lactoglobulin $(\mathrm{BlgB})$ is a small lipocalin (18.3 $\mathrm{kDa}$ ) that has ability to bind wide range of hydrophobic ligands. $\mathrm{B} \operatorname{lgB}$ could be re-engineered to gain high specificity towards selected compounds. A series of new $\mathrm{B} \operatorname{lgB}$ variants were design utilizing analysis of substitutions occurrence in structurally related lipocalins. Mutations were introduced to the protein binding site to investigate their impact on $\mathrm{B} \operatorname{lgB}$ binding properties and structural stability.

All lactoglobulin variants were produced in E.coli Origami $B(D E 3)$ and purified to homogeneity by ion-exchange and size-exclusion chromatography. New mutants and their complexes with ligands were crystallized by hanging-drop vapour diffusion method. X-ray diffraction data were collected on SuperNova diffractometer. Structures were solved by molecular replacement.

Crystal structures of new $\mathrm{B} \operatorname{lgB}$ variants revealed that some amino acid replacements in the binding site especially in positions 56, 58, 92, 105 and 107 stabilize protein structure. It was also observed that mutations in position 56 and 105 affect mode of binding of aromatic or aliphatic compounds by altering the shape of the hydrophobic $\beta$-barrel interior. Modifications introduced at the binding site entrance where most of polar residues are located, did not affect significantly ligand binding mode but seem to destabilize protein structure and alter interactions in the crystalline phase.

Results of structural and in-solution studies of new lactoglobulin variants revealed that $\mathrm{B} \operatorname{lgB}$ affinity to compounds possessing aliphatic and aromatic fragments can be modified mostly by modelling the shape of binding pocket. Additional polar residues introduced to the region of binding, that act as hydrogen bond donors and acceptors, play a secondary role in increasing lactoglobulin affinity to selected ligands.

This study was supported by the Polish National Science Centre, grant number 2012/05/B/ST5/00278.

Keywords: lipocalin, lactoglobulin, mutations
MS9-P14 Crystallographic fragment screening: challenges, opportunities and lessons learned

Andreas Heine $^{1}$, Johannes Schiebel ${ }^{1}$, Nedyalka Radeva ${ }^{1}$, Alexander Metz ${ }^{1}$, Franziska U. Huschmann ${ }^{1,2}$, Monika Ühlein ${ }^{2}$, Karine Sparta ${ }^{2}$, Manfred S. Weiss ${ }^{2}$, Uwe Mueller ${ }^{2}$, Gerhard Klebe $^{1}$

1. Institute of Pharmaceutical Chemistry, Philipps-University Marburg, Marburg, Germany

2. Helmholtz-Zentrum Berlin für Materialien und Energie, Macromolecular Crystallography, Berlin, Germany

email: heinea@staff.uni-marburg.de

Nowadays, fragment screening is an established method for lead development in pharmaceutical drug research. Library design and applied screening methods are critical for successful hit identification. We generated a small library, consisting of 364 compounds, which does not strictly adhere to the Astex rule of three often applied for library design. 1 The pepsin-like aspartyl protease endothiapepsin (EP) was selected for library validation. EP is a model system for proteins involved in serious diseases such as malaria (plasmepsins), hypertension (renin) and Alzheimer's disease ( $\beta$-secretase) and, therefore, can aid in further drug development. Fragments tend to exhibit only low affinity toward their target protein due to their limited size. This hampers hit identification and, therefore, often multiple screening methods are applied. We used a biochemical as well as various biophysical assays as pre-screening methods. Surprisingly, the results of the different screening methods showed only limited overlap. Thus, we decided that crystallographic screening of the entire library is ultimately required to identify all fragments that bind to EP. In any case, the resulting structural information is the basis for further synthesis by medicinal chemists. Crystallographic screening of an entire library requires a steady supply of crystals, reproducible soaking conditions and a sufficient amount of beamtime. The latter was provided by the HZB BESSY II MX beamlines, ${ }^{2}$ in combination with automation in initial data processing and refinement. More than 60 fragment complex structures were obtained which will be compared to results from other screening methods. The binding modes of the resulting fragments will be discussed and provide an ideal basis for further development of endothiapepsin inhibitors.

1. H. Köster, T. Craan, S. Brass, C. Herhaus, M. Zentgraf, L. Neumann, A. Heine, G. Klebe; A Small Nonrule of 3 Compatible Fragment Library Provides High Hit Rate of Endothiapepsin Crystal Structures with Various Fragment Chemotypes, J. Med. Chem. 54, 2011, 7784-7796.

2. U. Mueller, N. Darowski, M. R. Fuchs, R. Forster, M. Hellmig, K. S. Paithankar, S. Puhringer, M. Steffien, G. Zocher, M. S. Weiss; Facilities for macromolecular crystallography at the Helmholtz-Zentrum Berlin, J. Synchrotron Radiat. 19, 2012, 442-449.

Keywords: fragment-based drug design 